Check for updates

Cite this: RSC Adv., 2018, 8, 36049

\title{
A high precision length-based carbon nanotube ladder
}

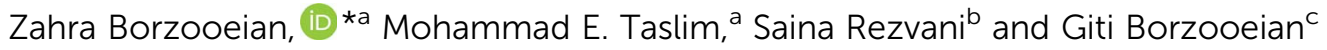

Today, carbon nanotubes manufacturers as well as users such as molecular electronics, nanomedicine, nano-biotechnology and similar industries are facing a major challenge: lack of length uniformity of carbon nanotubes in mass production. An effective solution to this major issue is the use of a lengthbased ladder. We are, for the first time, presenting such a valuable tool to determine the length purity. Our length-based carbon nanotubes ladder, containing a series of carbon nanotubes markers with different lengths, is made based on three combined techniques - bio-conjugation, sodium dodecyl sulfate polyacrylamide gel electrophoresis (SDS-PAGE), and silver staining. Creating an indicator using conjugation of a biomolecule with carbon nanotubes to make a carbon nanotubes ladder is a novel idea and a significant step forward for length-based carbon nanotubes separation. The very sensitive silver staining technique allows a precise visualization and quantification of the gel. This ladder with a pending patent by Northeastern University is an effective quality control tool when bulk quantities of nanotubes with a desirable length are manufactured.
\end{abstract}

Received 26th June 2018

DOI: $10.1039 / \mathrm{c} 8 \mathrm{ra} 05482 \mathrm{~g}$

rsc.li/rsc-advances

polyacrylamide gel electrophoresis (SDS-PAGE). Lysozyme was attached onto the carboxyl functionalized carbon nanotubes and horn sonication was used to create conjugated CNTs with different lengths. Creating an indicator for length-based separation of CNTs by the conjugation of biomolecules onto the nanotubes surfaces is a novel idea and a significant step forward in CNT-based science. Separation of bio-conjugated CNTs was performed using SDS-PAGE because of the chargeand size-dependent mobility of bio-conjugated CNTs under the influence of an applied electric field. Visualization with high resolution and quantification of nanotube fragments in the acrylamide gel, a challenge for a number of nano-tech researchers, was achieved using highly-sensitive silver staining. This ladder serves as a valuable quality-control tool when bulk quantities of CNTs with a specific length are produced. are impacted by the nanotube length, to name a few. Therefore, a primary requirement for any technique that separates and purifies nanotubes in a scalable and reproducible manner is the ability to perform a precise measurement of the nanotubes length..$^{18}$ To the best of these authors' knowledge, there exists no reliable quality control tool for precise and rapid measurement of nanotubes length and level of purity. Our proposed lengthbased ladder is intended for that purpose.

The ladder is created through the covalent conjugation of lysozyme onto CNTs followed by sodium dodecyl sulfate

\footnotetext{
${ }^{a}$ Department of Mechanical and Industrial Engineering, College of Engineering, Northeastern University, Boston, MA, USA. E-mail: z.borzooeian@northeastern.edu; borzooeianm@gmail.com

${ }^{b}$ Department of Computer Science, Worcester Polytechnic Institute, Worcester, MA, USA 'Department of Biology, Payamnoor, University of Esfahan, Iran
}

\section{Materials and methods}

\section{Conjugation of lysozyme onto SWCNTs}

Details of covalent conjugation of lysozyme (lyophilized chicken egg white lysozyme, EC 3.2.1.17, Inovatech, Inc. Abbotsford, BC, Canada) to the functionalized single-walled carbon nanotubes (SWCNT-COOH) with outer diameter of 1-2 nm (MKnano, Canada) were given in our previous work. ${ }^{19,20}$ Conjugation was achieved using carbodiimide method. ${ }^{21}$ One $\mathrm{mg}$ of CNTs was dispersed in one $\mathrm{mL}$ of MES buffer, $50 \mathrm{mM}, \mathrm{pH}$ 6.2, and was added to an equal volume of $400 \mathrm{mM}$ NHS in MES buffer. For coupling of NHS to the carboxylic groups on the surface of nanotubes, $20 \mathrm{mM}$ EDC was added to the mixture. The mixture was then stirred at $200 \mathrm{rpm}(30 \mathrm{~min})$ followed by sonication 
(MSE Ultrasonic Disintegrators, $150 \mathrm{~W}$, England) for $30 \mathrm{~min}$ and then centrifuged at $7000 \mathrm{rpm}$, three times, $15 \mathrm{~min}$ each to remove excess EDC and NHS. The enzyme solution which contained $10 \mathrm{mg}$ of lysozyme in $1 \mathrm{~mL}$ of phosphate buffer $(10 \mathrm{mM}$, $\mathrm{pH} 8$ ) was then added to the nanotubes solution. The final mixture was sonicated for $c a .1 \mathrm{~min}$ to re-disperse the SWCNTs. The solution was shaken in an orbital shaker at $200 \mathrm{rpm}$ at room temperature during the conjugation process. The conjugated lysozyme-SWCNTs solution was then centrifuged. To remove all nonspecifically adsorbed enzyme completely, the mixture was washed three times with triply distilled water and once with $1 \%(\mathrm{v} / \mathrm{v})$ Tween-20. Control enzyme-nanotube conjugates were prepared using the same procedure, only without using EDC and NHS.

\section{Characterization of conjugated lysozyme-SWCNTs}

The morphology of conjugated SWCNTs with lysozyme was compared with that of activated SWCNTs and pure lysozyme using scanning electron microscopy (SEM, S360 Oxford), TGA (TGA Q50), X-ray diffraction (XRD, D8, Advance, Bruker, axs) at $\lambda$ $=0.1542 \mathrm{~nm}$, and FTIR spectroscopy (Shimadzu FTIR 8300 spectrophotometer). To prepare three different ladders of SWCNTs, conjugated samples were sonicated for three time periods of 3, 7 and 10 minutes. $^{22}$

\section{SDS-PAGE and silver staining}

To prepare the gel stock solution $(30 \%, \mathrm{~m} / \mathrm{v})$, acrylamide $(29.2 \mathrm{~g})$ and Bis $(0.8 \mathrm{~g})$ were dissolved in $100 \mathrm{~mL}$ of water and filtered. The separating gel solution was made up of $10.0 \mathrm{~mL}$ of the gel stock solution, $10.0 \mathrm{~mL}$ of Tris- $\mathrm{HCl}$ (1.5 $\mathrm{mol} \mathrm{L}^{-1}$, $\left.\mathrm{pH} 8.80\right), 200-$ $800 \mu \mathrm{L}$ of $\left(\mathrm{NH}_{4}\right)_{2} \mathrm{~S}_{2} \mathrm{O}_{8}(10 \% \mathrm{~m} / \mathrm{v})$ and $0.4 \mathrm{~g}$ of SDS, diluted with water to $40 \mathrm{~mL}$. The stacking gel was prepared by mixing $1.33 \mathrm{~mL}$ of the gel stock solution with $2.5 \mathrm{~mL}$ Tris- $\mathrm{HCl}$ (0.5 mol L $\left.{ }^{-1}, \mathrm{pH} 6.80\right)$ and $50 \mu \mathrm{L}\left(\mathrm{NH}_{4}\right)_{2} \mathrm{~S}_{2} \mathrm{O}_{8}(10 \%, \mathrm{~m} / \mathrm{v})$, and diluting with water to $10.0 \mathrm{~mL}$. Then TEMED $(10 \mu \mathrm{L})$ was added to the mixture. To remove any noncovalently-adsorbed enzyme, samples were washed several times with phosphate buffer (10 mM, pH 8). Electrophoresis buffer was made by dissolving Tris $(15.14 \mathrm{~g})$, glycine $(72.05 \mathrm{~g})$, and SDS $(5 \mathrm{~g})$ in $500 \mathrm{~mL}$ of distilled water. Solution's pH was adjusted to 8.30. The final gel which consisted of separating $(10.0 \% \mathrm{~m} / \mathrm{v})$ and stacking $(3.0 \%$, $\mathrm{m} / \mathrm{v}$ ) gels was made in a vertical polyacrylamide gel system. Sample volumes of $15 \mu \mathrm{L}$ were loaded on the gel. The silver staining procedure (Blum method ${ }^{23}$ ) consisted of several steps: fixation with methanol, acetic acid and paraformaldehyde solutions, washing with ethanol (50\% and 30\%) and $\mathrm{dd}_{2} \mathrm{O}$, sensitizing with $\mathrm{Na}_{2} \mathrm{~S}_{2} \mathrm{O}_{3} \cdot 5 \mathrm{H}_{2} \mathrm{O}$, washing with dd $\mathrm{H}_{2} \mathrm{O}$, impregnating with silver nitrate and paraformaldehyde solution, washing with $\mathrm{ddH}_{2} \mathrm{O}$, developing with $\mathrm{Na}_{2} \mathrm{CO}_{3}$, paraformaldehyde and $\mathrm{Na}_{2} \mathrm{~S}_{2} \mathrm{O}_{3} \cdot 5 \mathrm{H}_{2} \mathrm{O}$ solution, washing with $\mathrm{ddH}_{2} \mathrm{O}$, and ending reaction with a stop solution - methanol $50 \%$, and acetic acid $12 \%$.

\section{Image analysis techniques for length measurement}

Two methods, a manual approach using ImageJ, a NIH approved software, and semi-automated method MATLAB used to analyse the gel images. Using the distance of each band, in a given lane, from the center (reference point) of the well and the electrical voltage applied to the gel, mobility and consequently length of the CNTs were calculated using Usrey's equation. ${ }^{24} \mathrm{~A}$ MATLAB code was developed to measure the intensities and the distances automatically. The input to this code was the 8 bit inverted image and the output was the distance of each band from the center of the well and the average intensity of the pixels of each band.

\section{Results and discussion}

Carbodiimide method ${ }^{19,21,25}$ was used for bio-conjugation of lysozyme onto carboxylated carbon nanotubes surface. The results from SEM micrographs (Fig. 1a-d; before and after conjugation), TGA results (Fig. 2) used for evaluation of chemical functionalization of SWCNTs and XRD patterns (Fig. 3) and FTIR (Fig. 4) for evaluation of bio-conjugation and its chemical stability, confirmed the attachment of lysozyme onto the SWCNTs surface.

The SEM micrographs (Fig. 1a-d) show the pre and post size and morphology of the conjugated SWCNTs. An increase of about 89.5-95 $\mathrm{nm}$ in the wall thickness of the conjugated nanotubes is an indication of a successful conjugation.

Thermograms of pure and functionalized SWNT with $\mathrm{COOH}$ are shown in Fig. 2a and b, respectively. Loss of weight with temperature is due to the burning of the attached chemical entities to the carbon nanotubes or nanotubes decomposition at elevated temperatures. Weight losses in two temperature ranges of $213-225^{\circ} \mathrm{C}$ and $340-600{ }^{\circ} \mathrm{C}$ correspond to combustion of the covalently-linked - $\mathrm{COOH}$ and burning decomposition of the carbon nanotubes, respectively. As shown in part (a),
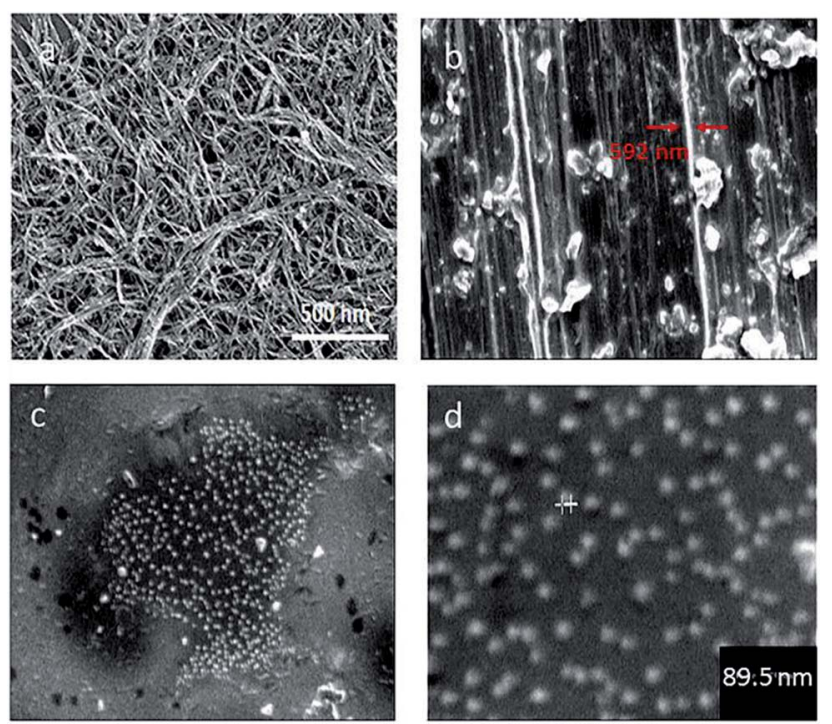

Fig. 1 SEM image of SWCNTs before and after conjugation. (a) SWCNTs before conjugation (b-d) SEM images of conjugated lysozyme-SWCNTs. (b) a magnification $1.50 \mathrm{k}$, diameter of the SWCNT bundle $\approx 592 \mathrm{~nm}$, (c) a magnification of $10 \mathrm{k}$, (d) a magnification $30.0 \mathrm{k}$, diameter of conjugated lysozyme-SWCNT $\approx 89.5 \mathrm{~nm}$. 

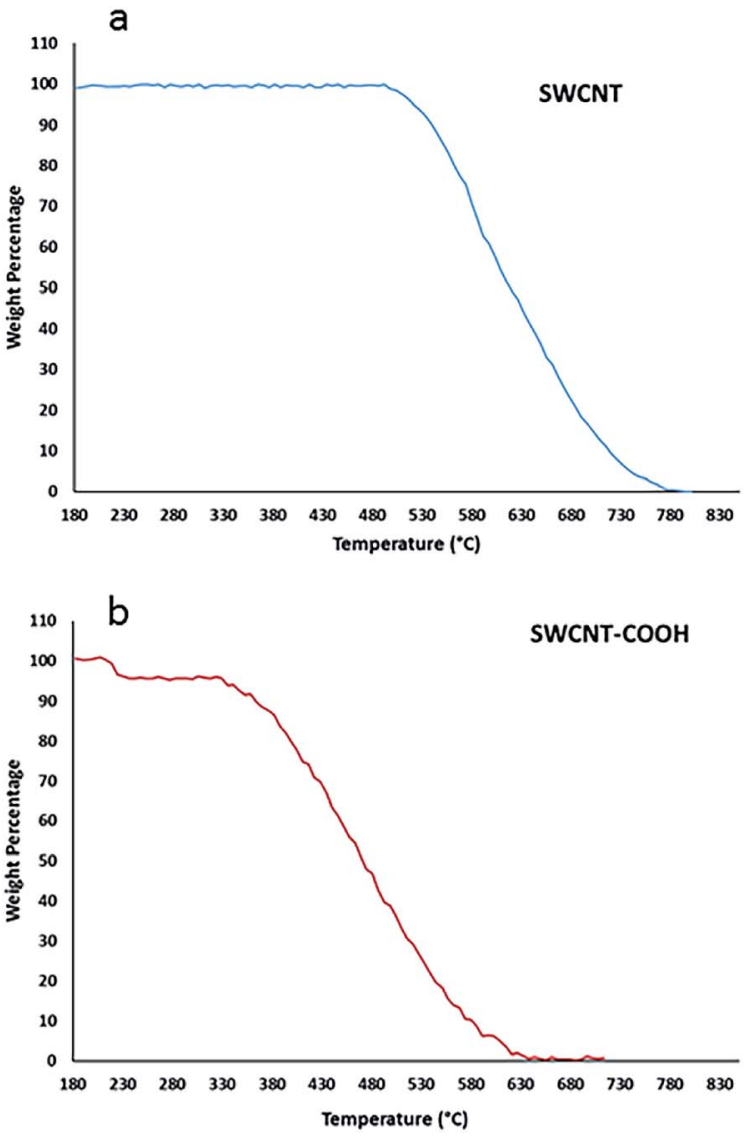

Fig. 2 TGA thermogram of pristine SWCNT ((a), blue) and functionalized SWCNT-COOH (b).

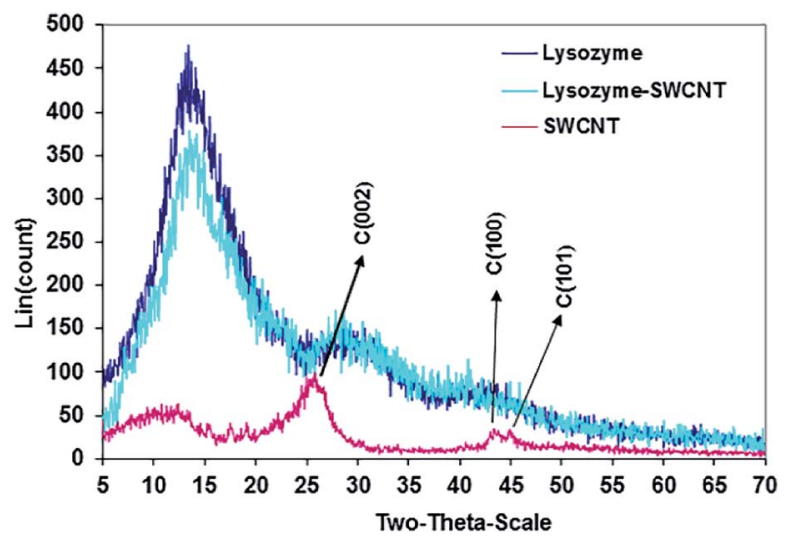

Fig. 3 X-ray diffraction (XRD) patterns of SWCNTs (red), lysozyme (dark blue), and conjugated lysozyme-SWCNTs (light blue).

SWCNT is thermally stable up to a temperature of about $500{ }^{\circ} \mathrm{C}$ indicating that it has no attached functional groups.

Detailed description of XRD results in Fig. 3 are presented in our earlier work. ${ }^{22}$ However, we shall refer to some important aspects of the results here. SWNCTs peak carbon planes are (002), (100) and (101). The Characteristic peaks of both free and conjugated lysozyme are the same and occur at $2 \theta$ positions 14.0, 30.0 and 42.0. The identical XRD patterns confirm the

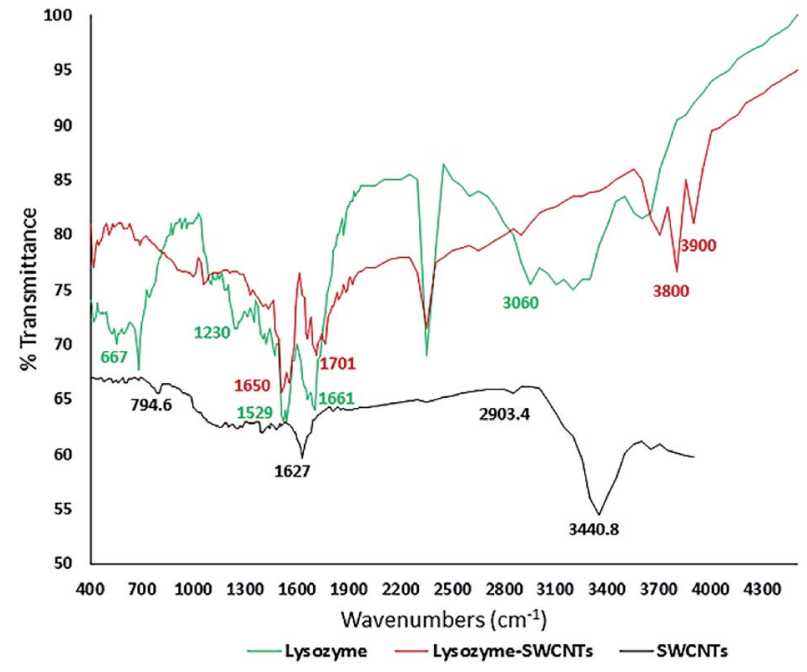

Fig. 4 FTIR spectra showing amide bonds in the conjugated lysozyme-SWCNTs. FTIR spectrum for lysozyme (green), the SWCNTs (black) and conjugated lysozyme-SWCNT (red).

adsorption or absorption of lysozyme onto SWCNTs with no lysozyme phase change.

The results of XPS analysis of the atomic concentrations of oxygen and nitrogen in SWCNT, SWCNT-COOH, and lysozymeSWCNT are presented in Table 1. The higher oxygen content for the SWCNT-COOH, compared with the pristine carbon nanotubes, confirms the presence of carboxyl groups on the surface of the nanotubes. The significant increase in both surface oxygen and nitrogen contents in conjugated lysozyme-SWCNTs indicates the covalent bonding between carboxyl-functionalized SWCNTs and lysozyme. The amount of immobilized enzyme was $1.1 \mathrm{mg} \mathrm{mg}^{-1}$ measured by elemental analysis of the activated carbon nanotubes and the lysozyme-SWNTs conjugates.

\section{FTIR analyses}

The amide linkages between the amino acid residues in polypeptides and proteins are detected in the FTIR diagrams. ${ }^{26}$ The covalent immobilization of polypeptides/proteins are studied by detecting the amide types I and II bands in the FTIR spectra which indicate the conformational changes in the protein secondary structure. ${ }^{27}$ Fig. 4 shows the FTIR spectra for pure lysozyme (green), pure SWCNTs (black), and lysozyme-SWCNT (red).

Chemical functionalization of SWCNTs with $-\mathrm{COOH}$ groups are confirmed by the position of two absorption peaks at $1627.8 \mathrm{~cm}^{-1}$ and $3440.8 \mathrm{~cm}^{-1}$ (black curve). The wide and strong $\mathrm{NH}_{3}$ stretching band of $2950-2600 \mathrm{~cm}^{-1}$ in the enzyme spectrum is the amino acid characteristic. The plateau region

Table 1 Atomic concentrations of SWCNT, SWCNT-COOH and lysozyme-SWCNT from XPS experimental data

\begin{tabular}{lll}
\hline Nanomaterial & $\begin{array}{l}\text { Atomic concentration } \\
\text { of oxygen }(\%)\end{array}$ & $\begin{array}{l}\text { Atomic concentration } \\
\text { of nitrogen }(\%)\end{array}$ \\
\hline
\end{tabular}

$\begin{array}{lll}\text { SWCNT } & 0 & 0\end{array}$

SWCNT-COOH $12 \quad 0$

Lysozyme-SWCNT $18 \quad 14$ 
a

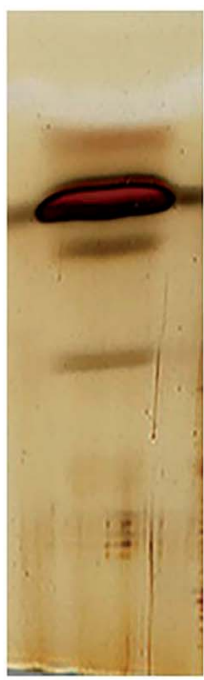

b

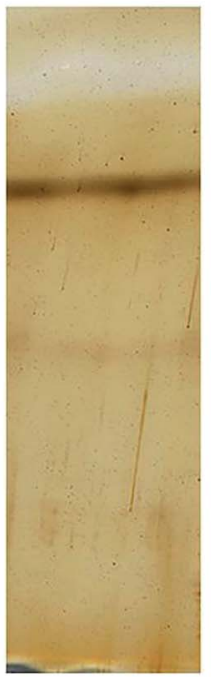

C

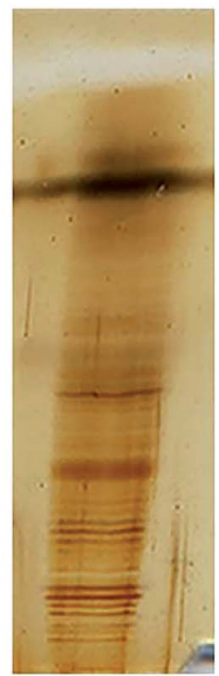

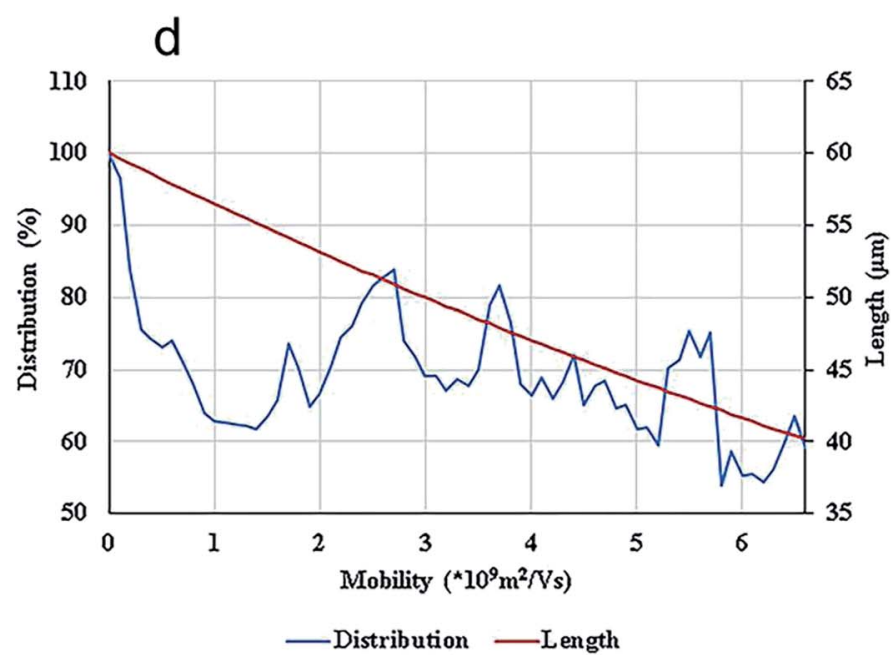

Fig. 5 SDS-PAGE electrophoresis and silver staining of lysozyme. (a), SWCNT (b), and ladder/conjugated lysozyme-SWCNT (c). Distribution and the lengths of SWCNT fragments (d).

near the band of 2222-2000 $\mathrm{cm}^{-1}$ corresponds to a combined bending vibration and torsional oscillation of the asymmetrical $\mathrm{NH}_{3}{ }^{+}{ }^{28}$ A weak bending band of asymmetric $\mathrm{NH}_{3}{ }^{+}$around $1661 \mathrm{~cm}^{-1}$ and a rather strong symmetric bending band around $1529 \mathrm{~cm}^{-1}$ are also observed. The $3600 \mathrm{~cm}^{-1}$ and $1230 \mathrm{~cm}^{-1}$ peaks represent the stretching of the $\mathrm{N}-\mathrm{H}$ and $\mathrm{C}-\mathrm{N}$ groups in the amine groups, respectively. Looking at the lysozymeSWCNTs spectrum, the disappearance of the peaks is due to the formation of amide bonds between the carboxyl groups of functionalized SWCNTs and amine groups of the enzyme. Stretching vibration mode of $\mathrm{C}=\mathrm{O}$ creates the $1650 \mathrm{~cm}^{-1}$ peak and stretching of the $\mathrm{N}-\mathrm{H}$ groups in the amide group creates the $3800 \mathrm{~cm}^{-1}$ and $1650 \mathrm{~cm}^{-1}$ peaks. In conclusion, the covalent bonding in the conjugation process was confirmed by the FTIR analyses, comparing the amide linkages between the free and conjugated lysozymes (peaks at $3800 \mathrm{~cm}^{-1}$ and $1650 \mathrm{~cm}^{-1}$ ).

Precise visualization of nanotube fragments in the acrylamide gel which is a challenge for a number of nano-tech researchers, was achieved using silver staining. Fig. 5 shows the migration of lysozyme (a), SWCNTs (b) and conjugated lysozyme-SWCNTs (c) fragments across the gel.

Stability of conjugation and sensitivity of silver staining technique are the reasons for such sharp bands in Fig. $5 \mathrm{c}$. The separation process of nano-carbon tubes in the gel based on their length is due to the following effects. Fragments of conjugated lysozyme-SWCNT with different lengths had different mobilities. Covalent attachment of lysozyme to carbon nanotubes gives rise to an intrinsic positive charge on any given individual nanotube or bundle affecting their mobilities. In other words, the degree of bioconjugation plays an important role in the separation process and net charge of fragments is directly proportional to the amount of conjugated lysozyme.

Depending on their length, each conjugated SWCNT moves differently through the gel matrix subjected to electrical field small CNT fragments will experience less resistance when passing through the pores in the gel, while larger ones have more difficulty. Therefore, the CNTs migrate different distances based on their length. Smaller CNTs travel farther down the gel, while larger ones remain closer to the point of loading. The velocity (mobility) of the charged CNT fragments is directly proportional to the electrical field $(E$, volts per $\mathrm{cm})$ and CNT fragments charge $(q)$, and inversely proportional to the frictional coefficient of the mass and shape of the fragment $(f){ }^{24}$

$$
V=\frac{E q}{f}
$$
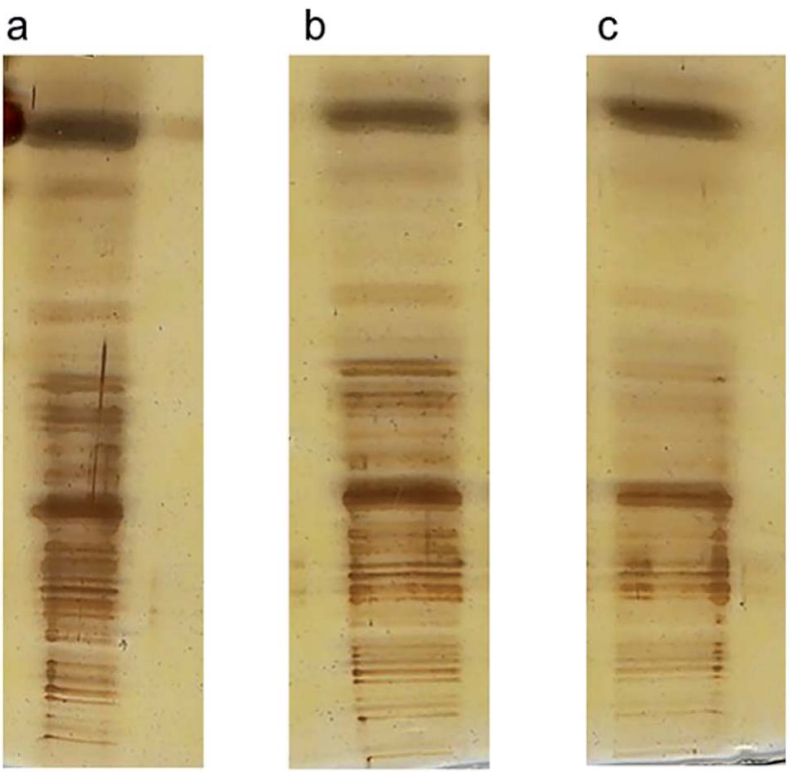

Fig. 6 Three ladders produced from 3 (a), 7 (b), and 10 (c) min sonication time. It is clear the conjugated SWCNTs fragments are separated based on their lengths. 
Since the gel acts like a sieve and retains the larger nanotubes while allowing the smaller ones pass through, the frictional coefficient is a representation of the level of resistance that the SWCNT fragments face as they pass through the pores of the gel. The SWCNT fragment length is also a key factor in its mobility in the gel matrix. In view of eqn (1), one will then have: mobility $=($ voltage $)($ charge $) /($ length $)$.

To summarize, during gel electrophoresis, the mobility of a SWCNT fragment is primarily a function of its charge/length ratio.

Usrey's eqn (2), relating the fragment length to the intensity of the bands of the lanes is used for the calculation of the length distribution of the conjugated SWCNTs.

$$
L=d \times \exp \left(\left[\frac{3 \pi \mu \eta}{(q(d) \times e)}-2 \ln (2)+1\right]\right)
$$

a
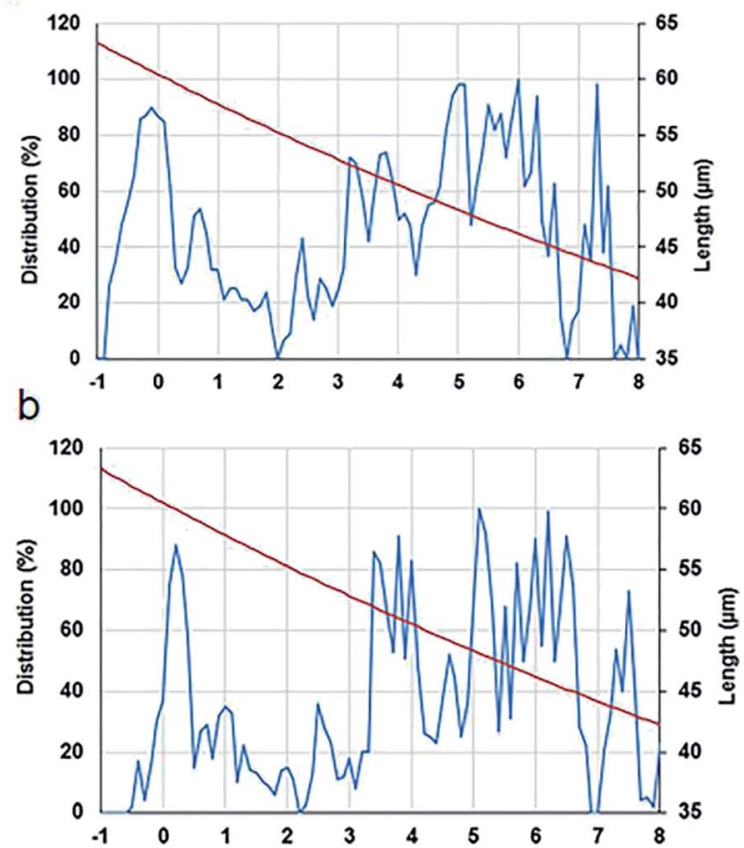

C

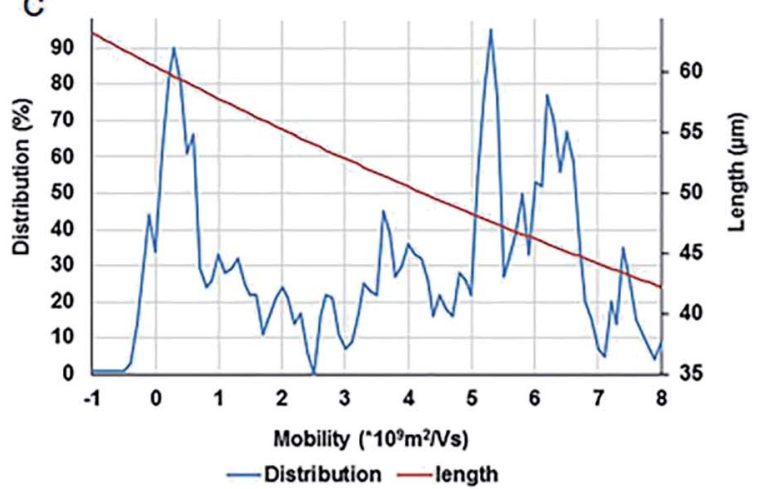

where $d=89.0 \pm 0.2(\mathrm{~nm})$ is the average diameter of each CNT and $\eta=$ viscosity $=1.25$ (Pa s), $q(d)$ is calculated according to Usrey $e t a l$. and $e$ is the electron charge. ${ }^{24}$

After an analysis of gel images using ImageJ, experimental data were obtained in the form of mobility distribution (number of nanotubes as a function of mobility). Fig. 5a-c show silver staining of free lysozyme (a), SWCNTs (b), and conjugated lysozyme-SWCNTs (c), and Fig. 5d shows that SWCNTs of various lengths are present in the population for each experimental electrophoretic mobility value.

To validate our method of creating CNT ladders, we made three different ladders of conjugated SWCNT fragments with different lengths, produced from sonication intervals of 3, 7, and $10 \mathrm{~min}$ (Fig. 6a-c, respectively). Conjugated lysozymeSWCNT fragments of different lengths showed different mobilities.

To calculate the length of CNTs, two methods were used. In the first method, a computer program was developed in MATLAB d

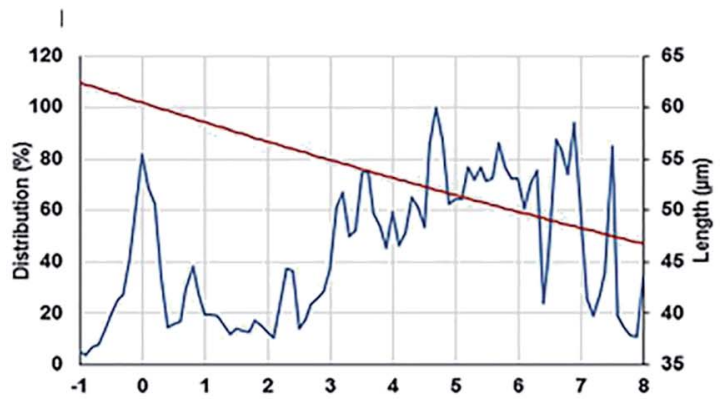

e

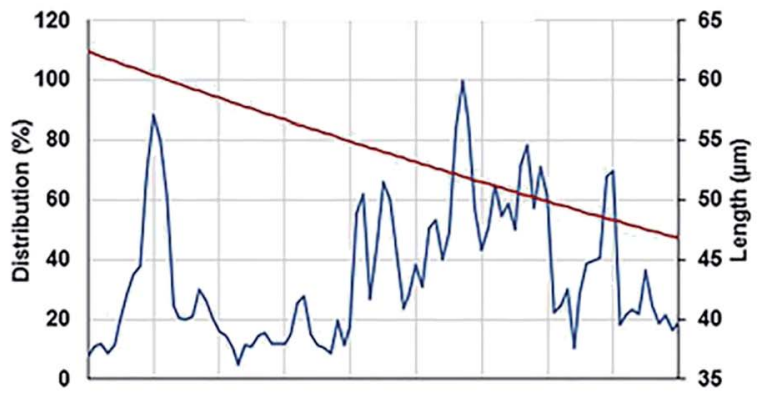

f

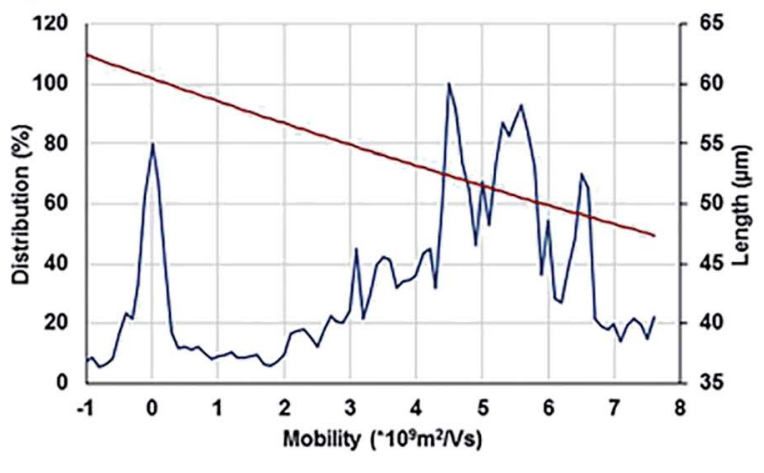

Fig. 7 Distribution and the lengths of SWCNT fragments in two methods (ImageJ, (a) through (c) \& MATLAB, (d) through (f)) after sonication time at 3 min ( $a$ and d), 7 min (b and e), and 10 min (c and f). 

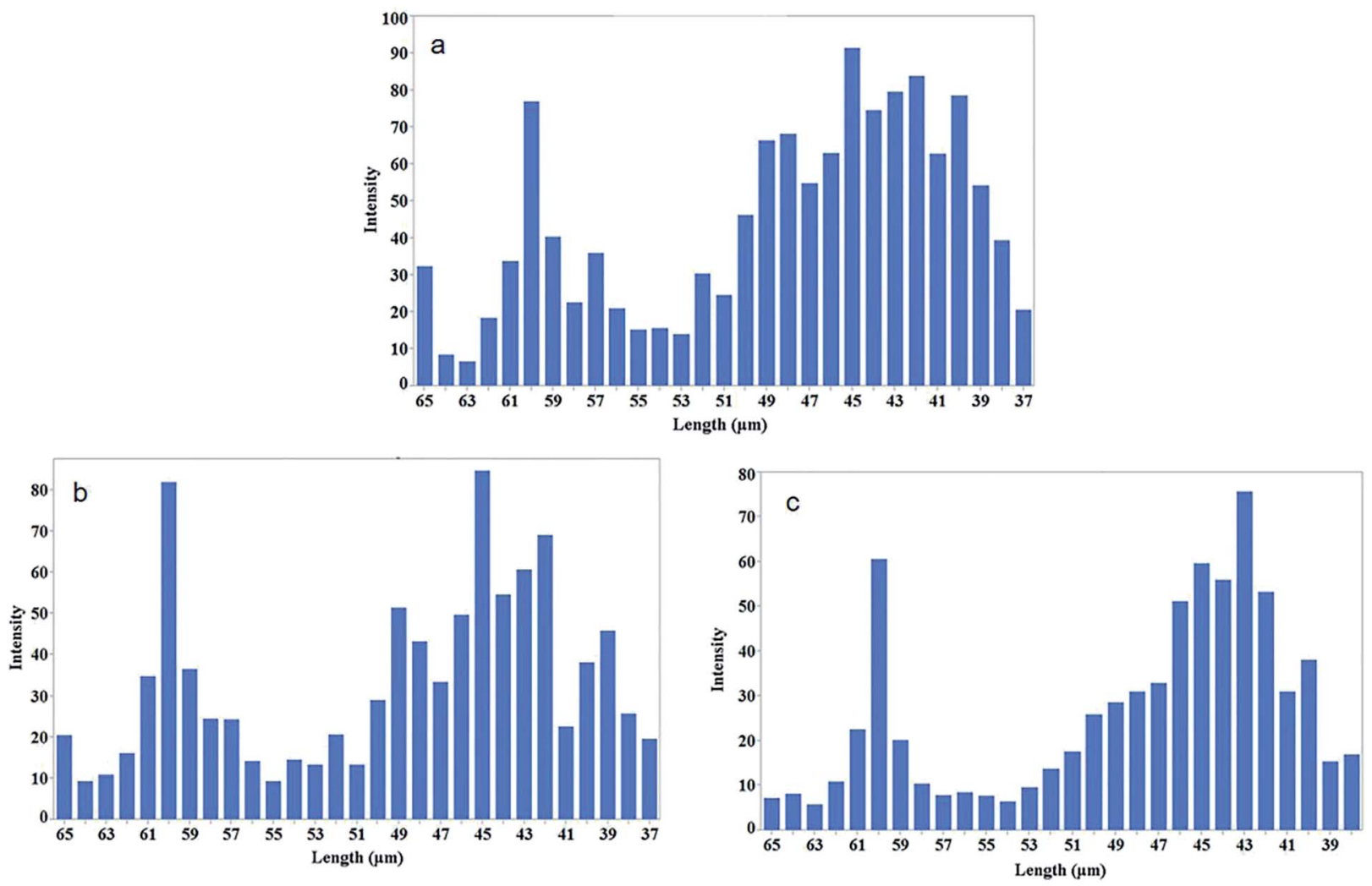

Fig. 8 Length distribution of the conjugated SWCNTs after sonication time at $3 \mathrm{~min}(\mathrm{a}), 7 \mathrm{~min}(\mathrm{~b})$, and $10 \mathrm{~min}$ (c). The intensity of the CNTs at each ladder is plotted versus length of CNTs calculated from eqn (1).

that subtracts the background, selects three lines on each lane of the gel and averages the signal (intensities of the bands) at each distance from the center of the wells. In the second method, ImageJ is used to calculate the same parameter using a narrow rectangle along each lane from the well to the bottom of the gel. These methods generated similar results (as shown in Fig. 7) that are in concordance with the visual evaluations.

After an analysis of gel images, experimental data were obtained in the form of mobility distribution (number of nanotubes as a function of mobility). The corresponding nanotube length of CNTs for every ladder were calculated from the Usray's formula (1). Fig. 7 shows the distribution and lengths of SWCNT fragments determined by two methods (ImageJ, Fig. 7a through Fig. 7c) \& (MATLAB Fig. 7d through Fig. 7f) for sonication intervals of $3 \mathrm{~min}$ (Fig. 7a and 7d), $7 \mathrm{~min}$ (Fig. 7b and 7e), and $10 \mathrm{~min}$ (Fig. $7 \mathrm{c}$ and $7 \mathrm{f}$ ). A key factor in our length-based separation technique is the change of surface charge density of nanotubes due to bio-conjugation and the number of attached biomolecules.

Fig. 8 shows the intensity of conjugated carbon nanotubes of different lengths for three different sonication intervals. Lengths are determine form the Usrey's eqn (1).

In conclusion, for the first time, taking advantage of conjugation of biomolecules onto CNT surfaces, a length-based CNT ladder is presented that can serve as a much-needed quality control tool for manufacturing bulk quantities of carbon nanotubes of specified lengths.

\section{Conclusions}

We have, for the first time, presented a valuable tool to determine the carbon nanotube length purity. Our lengthbased CNT ladder, containing a series of CNT markers with different lengths, is made by the combination of the following three techniques - bio-conjugation, SDS-PAGE, and silver staining. Creating an indicator using conjugation of a biomolecule with carbon nanotubes to make a CNT ladder is a novel idea and a significant step forward for length-based carbon nanotube separation. The very sensitive silver staining technique creates a high-resolution image, observable with naked eye and a precise quantification of the nanotubes. It further obviates the need for any further testing such as Raman spectroscopy. This ladder is an effective quality control tool when bulk quantities of nanotubes with a desirable length are manufactured.

\section{Conflicts of interest}

There are no conflicts to declare.

\section{Abbreviations}

SWCNT MES
Single-walled carbon nanotubes

2-( $N$-Morpholino)ethane sulfonic acid 
EDC

$N$-Ethyl- $N^{\prime}$-(3-(dimethyl amino)propyl) carbodiimide hydrochloride

NHS

Tris

Bis

SDS

TEMED

2ME

Bromophenol

Blue

\section{References}

1 E. G. Rakov, Russ. Chem. Rev., 2000, 69, 35-52.

2 F. Hennrich, S. Lebedkin, S. Malik, J. Tracy, M. Barczewski, H. Rösner and M. Kappes, Phys. Chem. Chem. Phys., 2002, 4, 2273-2277.

3 S. Mohsenian, J. Fathi and B. Shokri, Appl. Phys. A: Mater. Sci. Process., 2018, 124, 546.

4 S. Hermann, B. Pahl, R. Ecke, S. E. Schulz and T. Gessner, Microelectron. Eng., 2010, 87, 438-442.

5 R. H. Baughman, A. A. Zakhidov and W. A. de Heer, science, 2002, 297, 787-792.

6 M. S. Arnold, M. O. Guler, M. C. Hersam and S. I. Stupp, Langmuir, 2005, 21, 4705-4709.

7 J. Kong, N. R. Franklin, C. Zhou, M. G. Chapline, S. Peng, K. Cho and H. Dai, science, 2000, 287, 622-625.

8 A. Bachtold, P. Hadley, T. Nakanishi and C. Dekker, Science, 2001, 294, 1317-1320.

9 Z. Yao, H. W. C. Postma, L. Balents and C. Dekker, Nature, 1999, 402, 273-276.

10 A. Jones, T. Bekkedahl and C. Kiang, Nature, 1997, 386, 377. 11 P. Chen, X. Wu, J. Lin and K. Tan, Science, 1999, 285, 91-93. 12 C. Liu, Y. Fan, M. Liu, H. Cong, H. Cheng and M. S. Dresselhaus, Science, 1999, 286, 1127-1129.
13 H. He, L. A. Pham-Huy, P. Dramou, D. Xiao, P. Zuo and C. Pham-Huy, BioMed Res. Int., 2013, 2013, 578290.

14 S. K. Doorn, R. E. Fields, H. Hu, M. A. Hamon, R. C. Haddon, J. P. Selegue and V. Majidi, J. Am. Chem. Soc., 2002, 124, 3169-3174.

15 X. Wang, Q. Jiang, W. Xu, W. Cai, Y. Inoue and Y. Zhu, Carbon, 2013, 53, 145-152.

16 A. D. Franklin and Z. Chen, Nat. Nanotechnol., 2010, 5, 858862.

17 B. Singh, K. Saini, V. Choudhary, S. Teotia, S. Pande, P. Saini and R. Mathur, J. Nanopart. Res., 2014, 16, 1-11.

18 N. Komatsu and F. Wang, Materials, 2010, 3, 3818-3844.

19 Z. Borzooeian, A. Safavi, M. Hossain Sheikhi, M. Aminlari and M. Mahdi Doroodmand, J. Exp. Nanosci., 2010, 5, 536547.

20 Z. Borzooeian, M. Taslim, G. Borzooeian, O. Ghasemi and M. Aminlari, RSC Adv., 2017, 7, 48692-48701.

21 P. Asuri, S. S. Bale, R. C. Pangule, D. A. Shah, R. S. Kane and J. S. Dordick, Langmuir, 2007, 23, 12318-12321.

22 Z. Borzooeian, O. Ghasemi, S. Rezvani, G. Borzooeian and A. Nourbakhsh, PLoS One, 2018, DOI: 10.1371/ journal.pone.0197972.

23 H. Blum, H. Beier and H. J. Gross, Electrophoresis, 1987, 8, 93-99.

24 M. L. Usrey, N. Nair, D. E. Agnew, C. F. Pina and M. S. Strano, Langmuir, 2007, 23, 7768-7776.

25 Z. Borzooeian, G. Borzooeian, O. Ghasemi and M. Aminlari, RSC Adv., 2017, 7, 48692.

26 A. Dong, P. Huang and W. S. Caughey, Biochemistry, 1992, 31, 182-189.

27 A. Dong, B. Caughey, W. S. Caughey, K. S. Bhat and J. E. Coe, Biochemistry, 1992, 31, 9364-9370.

28 R. M. Silverstein, F. X. Webster, D. J. Kiemle and D. L. Bryce, Spectrometric identification of organic compounds, John wiley \& sons, 2014. 